\title{
Aspirin decreases the risk of depression in older men with high plasma homocysteine
}

\author{
OP Almeida ${ }^{1,2,3}$, L Flicker ${ }^{2,4,5}$, BB Yeap ${ }^{4,6}$, H Alfonso $^{1,2}, \mathrm{~K} \mathrm{McCaul}^{2}$ and GJ Hankey ${ }^{4,7}$
}

High total plasma homocysteine (tHcy) is associated with increased risk of cardiovascular events and depression. Consumption of B-vitamins (B6, B9 and B12) reduces tHcy by about $15 \%$, but has equivocal effects on these health outcomes, suggesting that this relationship is either not causal or is confounded by other factors. The results of recent randomized trials suggest that antiplatelet therapy may confound these associations. This cross-sectional study assessed 3687 men aged 69-87 years for history of clinically significant depression (Geriatric Depression Scale 15 items $\geqslant 7$ ) or a recorded diagnosis of depression in the Western Australian Data Linkage System, and collected information on the use of aspirin, B-vitamins and antidepressant medication, along with age, education, living arrangements, smoking history and medical comorbidity as assessed by the Charlson index. Participants donated a blood sample for the measurement of tHcy, and concentrations $\geqslant 15 \mu$ mol I ${ }^{-1}$ were considered high. Five hundred and thirteen (13.9\%) men showed evidence of depression, and of those $31.4 \%$ had high tHcy, $41.5 \%$ were using aspirin, $6.8 \%$ were consuming B-vitamins. Multivariate logistic regression showed that high thcy was associated with increased odds of depression (odds ratio $(\mathrm{OR})=1.60,95 \%$ confidence interval $(\mathrm{Cl})=1.20-2.14$ ), as was the use of $\mathrm{B}$-vitamins $(\mathrm{OR}=1.95,95 \% \mathrm{Cl}=1.21-3.13)$. There was a significant interaction between high tHcy and aspirin use $(\mathrm{OR}=0.57$, $95 \% \mathrm{Cl}=0.36-0.91)$, but not between high tHcy and $\mathrm{B}$-vitamin use $(\mathrm{OR}=0.80,95 \% \mathrm{Cl}=0.26-2.46)$. The analyses were adjusted for smoking status, Charlson index and use of antidepressants. The results of this study indicate that older men with high tHcy who use aspirin have lower risk of depression, and suggest that antiplatelet therapy may be an effective preventive or management strategy for these cases. Randomized trials are required to confirm the antidepressant effect of aspirin in people with high tHcy.

Translational Psychiatry (2012) 2, e151; doi:10.1038/tp.2012.79; published online 14 August 2012

\section{Introduction}

Depression is a common and disabling condition that affects people of all ages, ${ }^{1,2}$ and about $10 \%$ of those aged 60 years or older. ${ }^{3}$ Currently available antidepressant therapies lead to a significant improvement in symptoms, although nearly half of those treated fail to respond or respond only partly. ${ }^{4,5}$ Increasing age has been implicated in treatment resistance, ${ }^{4}$ as has the presence of cerebrovascular disease,${ }^{6}$ although such knowledge has not as yet led to more effective forms of treatment. $^{7}$

Genetic epidemiological studies have shown that high total plasma homocysteine (tHcy) increases the risk of depression, ${ }^{8,9}$ possibly due to its association with higher risk of cardiovascular events in general, ${ }^{10}$ and strokes in particular. ${ }^{11}$ Indeed, observational data suggest that should the link between high tHcy and depression be causal, about $15 \%$ of all cases of depression in later life could be avoided by reducing tHcy. ${ }^{12}$ The use of folic acid (vitamin B9) with or without vitamins $B 12$ and B6 reduces thcy by about $15-20 \%,{ }^{13}$ but has equivocal effects on the risk-reduction of secondary cardiovascular events ${ }^{14}$ and of depression. ${ }^{15}$ A noteworthy exception originated from a secondary analysis of a randomized trial of B-vitamins, which found that post-stroke patients assigned treatment with $2 \mathrm{mg}$ of folic acid, and $25 \mathrm{mg}$ and $0.5 \mathrm{mg}$ of vitamins B6 and B12 experienced a 52\% (95\% confidence interval $(\mathrm{Cl}), 95 \% \mathrm{Cl}: 24-69 \%)$ reduction in the hazard of depression over 7 years compared with people treated with placebo. ${ }^{16}$ Taken together, these results suggest that clinical benefits of B-vitamins may only become apparent after their prolonged use (years rather than weeks) or, alternatively, that the lowering of tHcy by B-vitamins is insufficient to change the outcomes of patients.

High tHcy promotes endothelial dysfunction and facilitates platelet activation, ${ }^{17}$ suggesting that antiplatelet therapy could potentially minimize its atherogenic effects. Aspirin is the most widely used and effective antiplatelet treatment currently available, ${ }^{18}$ and a recent sub-analysis of the VITAmins TO Prevent Stroke (VITATOPS) trial suggested that aspirin overrides any beneficial effects of lowering tHcy with B-vitamin supplementation on the risk of cardiovascular events. ${ }^{19}$ Consequently, aspirin may be particularly helpful for people

${ }^{1}$ School of Psychiatry \& Clinical Neurosciences, University of Western Australia, Perth, WA, Australia; ${ }^{2}$ Western Australian Centre for Health \& Ageing, Centre for Medical Research, University of Western Australia, Perth, WA, Australia; ${ }^{3}$ Department of Psychiatry, Royal Perth Hospital, Perth, WA, Australia; ${ }^{4}$ School of Medicine and Pharmacology, University of Western Australia, Perth, WA, Australia; ${ }^{5}$ Department of Geriatric Medicine, Royal Perth Hospital, Perth, WA, Australia; ${ }^{6}$ Department of Endocrinology and Diabetes, Fremantle Hospital, Perth, WA, Australia and ${ }^{7}$ Department of Neurology, Royal Perth Hospital, Perth, WA, Australia

Correspondence: Professor OP Almeida, Western Australian Centre for Health \& Ageing (M573), Centre for Medical Research, University of Western Australia, 35 Stirling Highway, Crawley, Perth, WA 6009, Australia.

E-mail: osvaldo.almeida@uwa.edu.au

Keywords: ageing; aspirin; B-vitamins; cardiovascular disease; depression; homocysteine

Received 8 June 2012; revised 6 July 2012; accepted 8 July 2012 
with high tHcy. Furthermore, if high tHcy increases vulnerability to depression, ${ }^{8,9}$ one might expect treatment with aspirin to be associated with less depression among individuals with high tHcy. We aimed to test this hypothesis in a large population of elderly men.

\section{Materials and methods}

Participants and setting. This study used a populationbased sample of older men living in the Perth metropolitan area, the Health In Men Study (HIMS). Details regarding the recruitment of participants have been described elsewhere. ${ }^{20}$ Briefly, we recruited a community-representative sample of 19352 Australian men aged 65-85 years living in the Perth metropolitan area between 1996 and 1998, of whom 12203 completed the first assessment of HIMS. Five years later, 4247 of the 10940 surviving men agreed to complete a selfrating health assessment and donate a blood sample for testing (years 2001-2004). Of those, 560 showed evidence of cognitive impairment (Mini-Mental State Examination score $<24$ ) and were excluded from these analyses, leaving a study sample of 3687 participants aged 69-87 years.

The Human Research Ethics Committee of the University of Western Australia approved the study protocol and all men provided written informed consent to participate.

Outcome of interest: current or past clinically significant depression. Participants were asked to complete the 15-item Geriatric Depression Scale (GDS-15) and, a priori, those with a total score of 7 or more were considered to display clinically significant depressive symptoms at the time of assessment. This relatively high cut-point was chosen to ensure high specificity for the diagnosis of depression in this sample. ${ }^{21}$ In addition, we asked if a doctor had previously advised them that they had had depression (yes/no) and whether they had ever received treatment for an emotional disorder such as depression (yes/no). We considered that current or past clinically significant depression was present if men answered 'yes' to either of these two questions or if they had a GDS-15 score $\geqslant 7$ at the time of assessment. We chose this approach to define the outcome of interest of our study because of the recurrent nature of depression. ${ }^{22}$

Explanatory variables. We asked our men to list all medications they were consuming at the time of assessment, and used the World Health Organisation Anatomical Therapeutic Chemical classification system to determine whether participants were consuming antidepressants (codes N06A), B-vitamins (code B03B) or aspirin (codes B01AC06, M01BA03, N02BA01, N02BA51 and N02BA71) (World Health Organisation Collaborating Centre for Drug Statistics Methodology, Oslo, Norway, 2005). We also retrieved data on the use of aspirin that had been collected from these men between 1996 and 1998, when participants were asked: 'In the last month have you been taking aspirin tablets to prevent or treat heart disease?' Possible answers to this question were 'yes' or 'no'.

In addition, consenting men completed a self-report questionnaire that included items assessing demographic and clinical information. Age was calculated as the difference in years between the date of the assessment and the subject's date of birth, which we then used to create the following age groups: $69-75,75-79,80-84,85+$. Participants reported their country of birth (Australia or overseas), highest educational achievement and current living arrangements (living alone as opposed to living with others). Men were asked whether they had ever smoked (yes/no) and whether they were still smoking cigarettes, cigars or the pipe (yes/no). We used these answers to group them as 'never smokers', 'past smokers' and 'current smokers'.

We then retrieved administrative medical information from Western Australian Data Linkage System ${ }^{23,24}$ during the 10 years before assessment at HIMS to calculate the Charlson index. ${ }^{25}$ The index takes into account 17 common medical conditions that predict 1-year mortality: myocardial infarction, congestive heart failure, peripheral arterial disease, cerebrovascular disease, dementia, chronic pulmonary disease, connective tissue disease, ulcer disease, liver disease, diabetes (including diabetes with end organ damage), hemiplegia, renal disease, leukemia, lymphoma, other tumors, metastatic tumors and AIDS. Charlson and colleagues used adjusted relative risks to assign integer weights to these conditions within a composite index score that ranges from 0 to 37 . Coding algorithms to define comorbidities followed the procedures described by Quan et al. ${ }^{26}$ and scores were calculated using Stagg's Charlson's index Stata routine (StataCorp, College Station, TX, USA). Charlson weighted scores of 3 or more indicate the presence of significant medical comorbidity. ${ }^{25}$

Blood samples were collected between 0800 hours and 1030 hours. Plasma was prepared immediately following phlebotomy and stored at $-80^{\circ} \mathrm{C}$ until assayed. Biochemical assays were performed in the Department of Biochemistry, PathWest, Royal Perth Hospital, Western Australia. tHcy was measured by fluorescence polarization immunoassay on an IMx analyzer. ${ }^{27}$ We defined, a priori, that $\mathrm{tHcy} \geqslant 15 \mu \mathrm{mol} \mathrm{I}^{-1}$ was abnormally high. ${ }^{8}$

Statistical analyses. Data were analyzed with the statistical package Stata release 12.1 (StataCorp). We used descriptive statistics (mean, s.d. of the mean (s.d.), proportions) to summarize our data, and Pearson's $\chi^{2}$ statistic to test the distribution of demographic, lifestyle, clinical, medications and thcy variables according to group membership (Table 1). We then ran a series of multivariate logistic regression analyses using depression as the dependent variable; high tHcy, aspirin and use of B-vitamins as the independent variables (including interaction terms); and age, origin, education, living arrangements, smoking, the Charlson index and use of antidepressants as confounding variables. We used the backwards approach to remove confounding variables from the analyses if they were not independently associated with depression $(P>0.1)$ or contributed $<5 \%$ to the pseudo$R^{2}$ of the model. The risk ratio of these models corresponds to the odds ratio (OR) and their 95\% confidence interval $(95 \% \mathrm{Cl})$. Finally, we tested the goodness of fit of the model using the Hosmer-Lemeshow test.

Alpha was set at $5 \%$ and all probability tests reported in this paper were two-tailed. 
Table 1 Demographic, lifestyle and clinical characteristics of older men included in this study, as well as those with depression, thcy $\geqslant 15 \mu$ moll ${ }^{-1}$ or who were using aspirin and B-vitamins

\begin{tabular}{|c|c|c|c|c|c|c|c|c|c|}
\hline & \multirow{2}{*}{$\frac{\begin{array}{c}\text { Study population } \\
\mathrm{N}=3687\end{array}}{\mathrm{n}(\%)}$} & \multicolumn{2}{|c|}{$\begin{array}{c}\text { Depression } \\
\mathrm{N}=513\end{array}$} & \multicolumn{2}{|c|}{$\begin{array}{c}t H c y \geqslant 15 \mu \mathrm{mol} \\
\mathrm{N}=934\end{array}$} & \multicolumn{2}{|c|}{$\begin{array}{c}\text { Aspirin use } \\
\mathrm{N}=1511\end{array}$} & \multicolumn{2}{|c|}{$\begin{array}{c}\text { B-vitamin use } \\
\mathrm{N}=154\end{array}$} \\
\hline & & $\mathrm{n}(\%)$ & P-value & $\mathrm{n}(\%)$ & P-value & $\mathrm{n}(\%)$ & $\mathrm{P}$-value & $\mathrm{n}(\%)$ & P-value \\
\hline $\mathrm{tHcy} \geqslant 15 \mu \mathrm{moll}$ & $934(25.3)$ & $161(31.4)$ & 0.001 & - & - & $387(25.6)$ & 0.745 & $24(15.6)$ & 0.004 \\
\hline Aspirin use & $1511(41.0)$ & $213(41.5)$ & 0.789 & 387 (41.4) & 0.745 & - & - & $73(47.4)$ & 0.098 \\
\hline B-vitamin use & $154(4.2)$ & $35(6.8)$ & 0.001 & $24(2.6)$ & 0.004 & $73(4.8)$ & 0.098 & - & - \\
\hline Age in years & & & 0.061 & & $<0.001$ & & 0.001 & & 0.380 \\
\hline $69-74$ & $1849(50.1)$ & $230(44.8)$ & & $356(38.1)$ & & 709 (46.9) & & $67(43.5)$ & \\
\hline $75-79$ & $1218(33.0)$ & $183(35.7)$ & & $353(37.8)$ & & $512(33.9)$ & & $58(37.7)$ & \\
\hline $80-84$ & $531(14.4)$ & 87 (17.0) & & $181(19.4)$ & & $256(16.9)$ & & $24(15.6)$ & \\
\hline $85+$ & $89(2.4)$ & $13(2.5)$ & & $44(4.7)$ & & $34(2.2)$ & & $5(3.2)$ & \\
\hline Australian migrant & $1379(37.4)$ & $181(35.3)$ & 0.280 & $488(43.7)$ & $<0.001$ & 527 (34.9) & 0.009 & $69(45.1)$ & 0.045 \\
\hline Education: $\geqslant$ high school & $1861(50.5)$ & $268(52.2)$ & 0.399 & $460(49.3)$ & 0.391 & $772(51.2)$ & 0.515 & 87 (56.9) & 0.109 \\
\hline Living alone & $597(16.2)$ & $100(19.5)$ & 0.029 & $186(19.9)$ & $<0.001$ & $247(16.3)$ & 0.832 & $31(20.1)$ & 0.175 \\
\hline Smoking & & & $<0.001$ & & $<0.001$ & & 0.054 & & 0.557 \\
\hline Never & $1243(33.7)$ & $136(26.5)$ & & $258(27.7)$ & & 504 (33.4) & & $52(33.8)$ & \\
\hline Past & 2254 (61.2) & $340(66.3)$ & & $623(66.8)$ & & $944(62.5)$ & & $97(63.0)$ & \\
\hline Current & $188(5.1)$ & $37(7.2)$ & & $51(5.5)$ & & $62(4.1)$ & & $5(3.2)$ & \\
\hline Charlson index $\geqslant 3$ & $473(12.8)$ & $105(20.5)$ & $<0.001$ & $189(20.2)$ & $<0.001$ & $247(16.3)$ & $<0.001$ & $36(23.4)$ & $<0.001$ \\
\hline Antidepressant use & $220(6.0)$ & $153(29.8)$ & $<0.001$ & $69(7.4)$ & 0.034 & $112(7.4)$ & 0.002 & $12(7.8)$ & 0.329 \\
\hline
\end{tabular}

Abbreviation: tHcy, total plasma homocysteine.

The $P$-values were derived from unadjusted Pearson $\chi^{2}$ statistic and indicate the probability that the relevant exposures were associated with the respective group membership.

\section{Results}

The study sample included 3687 men aged 69-87 years (mean $=75.2$, s.d. $=4.1)$. Their demographic, lifestyle and clinical characteristics are summarized in Table 1. A total of 513 men $(13.9 \%)$ showed evidence of current $(n=165)$ or past depression. A larger proportion of men with than without depression was living alone and reported past or current smoking. They also had greater medical comorbidity, high tHcy, and were using B-vitamins and antidepressants more frequently than their non-depressed counterparts (Table 1).

Nine hundred and thirty-four men had tHcy $\geqslant 15 \mu \mathrm{moll}^{-1}$ (25.3\%). High tHcy was less frequent in the younger compared with older age groups, and more prevalent among migrants and those living alone. There was also an excess of past or current smokers among those with high tHcy, and a greater prevalence of significant medical comorbidity. Consumption of antidepressants and B-vitamins was more prevalent in men with than without high tHcy (Table 1).

Finally, aspirin use was less frequent among young than old older adults as well as migrants, but more frequent among those with significant comorbidity or who were using antidepressants. The use of B-vitamins was also more prevalent among migrants, men with significant comorbidity and those with high tHcy (Table 1).

Multivariate logistic regression showed that the odds of depression were $1.80(95 \% \mathrm{Cl}=1.39-2.35)$ for high tHcy, 1.18 $(95 \% \mathrm{Cl}=0.94-1.48)$ for aspirin, $2.12(95 \% \mathrm{Cl}=1.38-3.24)$ for B-vitamins, $0.60(95 \% \mathrm{Cl}=0.40-0.92)$ for the interaction between high tHcy and aspirin use and $0.60(95 \% \mathrm{Cl}=0.20$ 1.79) for the interaction between high tHcy and B-vitamin use. We then entered all variables listed in Table 1 into a regression model and removed them one at a time until the best possible fit was found. This parsimonious model included the following additional variables: smoking history, Charlson index grouping and use of antidepressants. High tHcy was associated with increased odds of depression $(\mathrm{OR}=1.60,95 \% \mathrm{Cl}=1.20-2.14)$, as was the use of $B$-vitamins $(\mathrm{OR}=1.95,95 \% \mathrm{Cl}=1.21-3.13)$. There was an interaction between high tHcy and aspirin use $(\mathrm{OR}=0.57,95 \% \mathrm{Cl}=0.36-0.91)$, but not between high tHcy and $\mathrm{B}$-vitamin use $(\mathrm{OR}=0.80,95 \% \mathrm{Cl}=0.26-2.46)$. Figure 1 illustrates these findings. The Hosmer-Lemeshow test assessing goodness of fit of the model confirmed that observed and expected outcomes did not differ significantly $\left(X^{2}[60]=60.98\right.$, $P=0.440$ ). We did not complete the analysis of the three-way interaction between high $\mathrm{tHcy}$, aspirin and B-vitamin because of a very large s.e.(that is, 5.39) of the OR caused by the presence of only three persons in the cell associated with depression.

\section{Discussion}

The results of this study confirmed that high tHcy is associated with an increased risk of depression and showed, for the first time, that the use of aspirin is associated a significantly lower risk among older men with high tHcy. These findings suggest that if high tHcy is a true cause of depression in later life, ${ }^{8,9,12}$ then aspirin could attenuate the incidence of depression among high-risk individuals in later life.

This study has several strengths. HIMS originated from a large community-representative sample of older men for whom a wealth of clinical and administrative data are available. $^{20}$ This enabled us to exclude participants who showed evidence of cognitive impairment (which is associated 


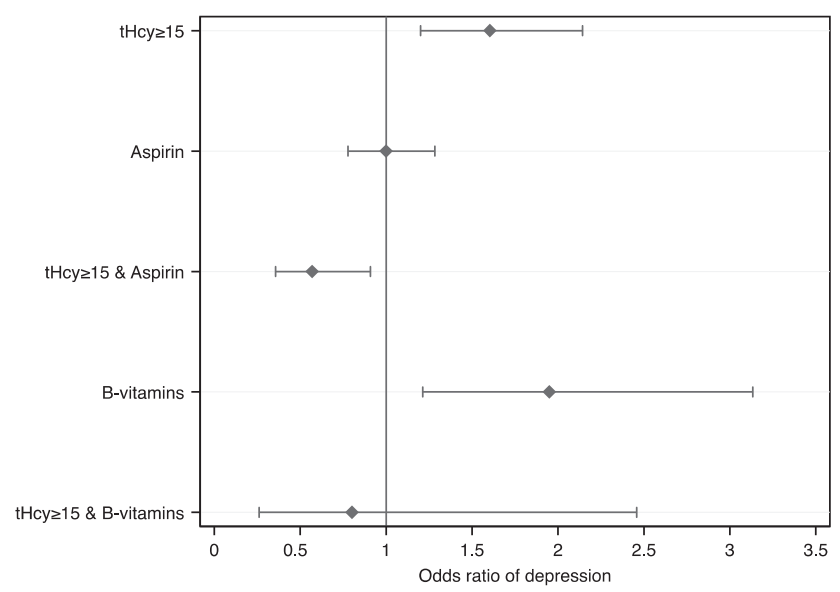

Figure 1 Odds ratio $(\mathrm{OR})$ of depression among older men according to their plasma concentration of homocysteine (tHcy $\geqslant 15 \mu \mathrm{mol}{ }^{-1}$ compared with $<15 \mu \mathrm{moll}^{-1}$ ), use of aspirin (yes compared with no), B-vitamins (yes compared with no) and the interaction between high plasma homocysteine and use of aspirin or B-vitamins. Older men with high tHcy using aspirin had lower odds of depression $(\mathrm{OR}=0.57 ; 95 \%$ confidence interval $(\mathrm{Cl})=0.36-0.91)$. Results of the multivariate logistic regression were further adjusted for smoking status, Charlson index grouping and use of antidepressants (other variables, including age, did not contribute significantly to the results of the model).

with both high tHcy and depression), ascertain the use of aspirin, vitamin B and antidepressants, gain access to tHcy, and adjust our analyses for factors known to influence tHcy, such as smoking and medical comorbidities. ${ }^{27}$ In addition, this investigation was sufficiently large to allow us to investigate interactions between exposures of interest and depression, although we lacked sufficient power to explore the three-way interaction between high tHcy, aspirin and vitamin B use because of the low prevalence of vitamin $B$ consumption in the sample. We acknowledge, however, that the crosssectional design of the study limits our ability to infer causality between the exposures and depression, and that reverse causality is possible. For example, a large proportion of older men with than without prevalent cardiovascular diseases might be in contact with health services, which would enhance the opportunistic diagnosis of depression and increase antidepressant treatment. Such men may also be more frequent users of aspirin and have high tHcy. Consequently, a lower proportion of men with high tHcy who are using aspirin would be expected to present with clinically significant depressive symptoms (because they are more likely to have health contacts and receive treatment). We have addressed this issue by retrieving Western Australian Data Linkage System data on depression and comorbidity recorded before the assessment for HIMS, and by adjusting our analyses for the use of antidepressants. The adjusted analyses indicate that our findings cannot be easily attributed to reverse causality or confounding due to the use of antidepressants.

We did not have access to information about the use of aspirin after the start of the follow-up period, and it is conceivable that any such changes could have had an effect on depression as an outcome. For example, if non-users with high tHcy initiated consumption of aspirin during follow-up, differences between the study groups would have been attenuated, and this would have biased the results toward the null hypothesis. A similar move toward the null hypothesis would have been associated with status change from user to non-user. Therefore, the 'antidepressant effect' of aspirin that we observed in this study cannot be easily explained by change in the consumption of aspirin during follow-up.

Our definition of 'depression' was not based on a structured psychiatric interview or use of accepted diagnostic guidelines, such as ICD-10 and DSM-IV-TR. Previous studies have shown that GDS-15 scores of 7 or greater have good face validity for the diagnosis of a depressive episode according to both the ICD and DSM systems, ${ }^{21}$ and the additional use of administrative data linkage would have ensured high specificity for cases, although sensitivity might have been suboptimal because people with less severe depressive symptoms might not come into contact with the Western Australian hospital system. The most likely consequence of such a bias would have been loss of power to explore associations with depression, possibly compromising our ability to examine the interaction between high tHcy and B-vitamin use on depression. In addition, we adjusted our analyses for factors known to increase tHcy and the risk of depression (such as smoking and comorbidities), but concede that our adjustments were not exhaustive and that residual confounding might explain some of the observed associations. Finally, we acknowledge that our data are limited to older men and we cannot be certain that similar results would have been found for women or younger adults.

A recent meta-analysis of over 200 genetic epidemiological studies that correlated strokes with tHcy and the methylene tetrahydrofolate reductase $\mathrm{C} 677 \mathrm{~T}$ polymorphism in 60000 people found that B-vitamin intake (particularly folate) is unlikely to reduce the risk of subsequent strokes in countries where folate supplementation of wheat is mandatory, ${ }^{28}$ suggesting that consumption of these vitamins might fail to reduce the health hazards associated with high tHcy if there is no overt vitamin deficiency. Furthermore, any possible cardiovascular benefit of supplementation with B-vitamins might be entirely overridden by the concomitant use of antiplatelet therapy (such as aspirin), ${ }^{19,29}$ suggesting that aspirin consumption may be a more helpful way of modifying the cardiovascular risk associated with high tHcy. This might explain our negative findings regarding the use of B-vitamins and risk of depression, even for older men with high tHcy. Indeed, it is possible that current optimistic views about the potential benefits of B-vitamins for the management of depression may lead to its overuse by people who are experiencing relevant symptoms, thus explaining the increased odds of depression that we observed among B-vitamin consumers (that is, prescription bias).

We have previously argued that the use of aspirin could potentially increase the risk of depression in later life because of bleeding and of other medical complications. ${ }^{30}$ That argument did not take into account the potential interaction between aspirin use and high $\mathrm{tHcy}$, which is a well-established risk factor for both cardiovascular diseases and depression. ${ }^{8,11}$ At this point, we can only speculate about the mechanisms by which aspirin modifies the risk of depression in older people with high tHcy. Aspirin might counteract the increased adhesive properties of platelets induced by high 
tHcy $^{17}$ or, alternatively, stimulate the release of monoamines that is partly compromised by dysfunction on one-carbon methylation associated with elevated tHcy. ${ }^{31,32}$ Regardless the physiological pathway, the results of this study indicate that older men with high tHcy who use aspirin have lower risk of depression, and suggest that antiplatelet therapy may be an effective preventive or management strategy for these cases. Randomized trials are required to confirm the antidepressant effect of aspirin in older persons with high tHcy. Future trials should test the efficacy of aspirin as an adjunctive therapy to standard antidepressants, as treatment with aspirin alone would be ethically questionable. No such concerns would limit the design of a placebo-controlled trial of aspirin to prevent depressive episodes in older people with high tHcy, particularly because homocysteine-lowering treatment with B-vitamins seems to have questionable impact on novel cardiovascular events and future depression. ${ }^{14,33}$

\section{Conflict of Interest}

The authors declare no conflict of interest.

Acknowledgements. This project was supported by competitive project grants from the National Health and Medical Research Council of Australia (NHMRC; numbers 279408, 379600, 403963, 513823 and 572594). We also thank research staff and study participants for their generous contribution.

Disclaimer. The NHMRC had no role in the design and conduct of the study; collection, management, analysis and interpretation of the data; or preparation, review or approval of the manuscript. OPA had full access to all the data in the study and takes responsibility for the integrity of the data and the accuracy of the data analysis.

\section{Author contributions}

Study concept and design: OPA. Acquisition of data: OPA, LF and GJH. Analysis of data: HA managed and extracted the data for analysis, and provided statistical advice; KMcC provided statistical advice; OPA performed all analyses. Drafting of the manuscript: OPA. Critical revision of the manuscript for important intellectual content: all authors.

1. Mathers CD, Vos ET, Stevenson CE, Begg SJ. The burden of disease and injury in Australia. Bull World Health Organ 2001; 79: 1076-1084.

2. Prince M, Patel V, Saxena S, Maj M, Maselko J, Phillips MR et al. No health without mental health. Lancet 2007; 370: 859-877.

3. Pirkis J, Pfaff J, Williamson M, Tyson O, Stocks N, Goldney R et al. The community prevalence of depression in older Australians. J Affect Disord 2009; 115: 54-61.

4. Tedeschini E, Levkovitz Y, lovieno N, Ameral VE, Nelson JC, Papakostas Gl. Efficacy of antidepressants for late-life depression: a meta-analysis and meta-regression of placebocontrolled randomized trials. J Clin Psychiatry 2011; 72: 1660-1668.

5. Fournier JC, DeRubeis RJ, Hollon SD, Dimidjian S, Amsterdam JD, Shelton RC et al. Antidepressant drug effects and depression severity: a patient-level meta-analysis. JAMA 2010; 303: 47-53

6. Baldwin R, Jeffries S, Jackson A, Sutcliffe C, Thacker N, Scott M et al. Treatment response in late-onset depression: relationship to neuropsychological, neuroradiological and vascular risk factors. Psychol Med 2004; 34: 125-136

7. Almeida OP. Vascular depression: myth or reality? Int Psychogeriatr 2008; 20: 645-652.

8. Almeida OP, McCaul K, Hankey GJ, Norman P, Jamrozik K, Flicker L. Homocysteine and depression in later life. Arch Gen Psychiatry 2008; 65: 1286-1294.

9. Lewis SJ, Lawlor DA, Davey Smith G, Araya R, Timpson N, Day IN et al. The thermolabile variant of MTHFR is associated with depression in the British Women's Heart and Health Study and a meta-analysis. Mol Psychiatry 2006; 11: 352-360.

10. Wald DS, Wald NJ, Morris JK, Law M. Folic acid, homocysteine, and cardiovascular disease: judging causality in the face of inconclusive trial evidence. BMJ 2006; 333 1114-1117.

11. Hankey GJ. Is homocysteine a causal and treatable risk factor for stroke? Lancet Neurol 2007; 6: 751-752.

12. Almeida OP, Flicker L, Norman P, Hankey GJ, Vasikaran S, van Bockxmeer FM et al. Association of cardiovascular risk factors and disease with depression in later life. Am J Geriatr Psychiatry 2007; 15: 506-513.

13. Flicker L, Vasikaran SD, Thomas J, Acres JM, Norman P, Jamrozik K et al. Efficacy of $B$ vitamins in lowering homocysteine in older men: maximal effects for those with B12 deficiency and hyperhomocysteinemia. Stroke 2006; 37: 547-549.

14. VITATOPS Trial Study Group. B vitamins in patients with recent transient ischaemic attack or stroke in the VITAmins TO Prevent Stroke (VITATOPS) trial: a randomised, doubleblind, parallel, placebo-controlled trial. Lancet Neurol 2010; 9: 855-865.

15. Taylor MJ, Carney SM, Goodwin GM, Geddes JR. Folate for depressive disorders: systematic review and meta-analysis of randomized controlled trials. J Psychopharmacol 2004; 18: 251-256.

16. Almeida OP, Marsh K, Alfonso H, Flicker L, Davis TM, Hankey GJ. B-vitamins reduce the long-term risk of depression after stroke: The VITATOPS-DEP trial. Ann Neurol 2010; 68: 503-510.

17. Stamler JS, Osborne JA, Jaraki O, Rabbani LE, Mullins M, Singel D et al. Adverse vascular effects of homocysteine are modulated by endothelium-derived relaxing factor and related oxides of nitrogen. J Clin Invest 1993; 91: 308-318.

18. Hankey GJ, Eikelboom JW. Antithrombotic drugs for patients with ischaemic stroke and transient ischaemic attack to prevent recurrent major vascular events. Lancet Neurol 2010; 9: 273-284.

19. Hankey GJ, Eikelboom JW, Yi Q, Lees KR, Chen C, Xavier D et al. Antiplatelet therapy and the effects of $B$ vitamins in patients with previous stroke or transient ischaemic attack: a post-hoc subanalysis of VITATOPS, a randomised, placebo-controlled trial. Lancet Neurol 2012; 11: 512-520

20. Norman PE, Flicker L, Almeida OP, Hankey GJ, Hyde Z, Jamrozik K. Cohort profile: the Health In Men Study (HIMS). Int J Epidemiol 2009; 38: 48-52.

21. Almeida OP, Almeida SA. Short versions of the geriatric depression scale: a study of their validity for the diagnosis of a major depressive episode according to ICD-10 and DSM-IV. Int J Geriatr Psychiatry 1999; 14: 858-865.

22. Alexopoulos GS. Depression in the elderly. Lancet 2005; 365: 1961-1970.

23. Holman CD, Bass AJ, Rosman DL, Smith MB, Semmens JB, Glasson EJ et al. A decade of data linkage in Western Australia: strategic design, applications and benefits of the WA data linkage system. Aust Health Rev 2008; 32: 766-777.

24. Holman CD, Bass AJ, Rouse IL, Hobbs MS. Population-based linkage of health records in Western Australia: development of a health services research linked database. Aust N ZJ Public Health 1999; 23: 453-459.

25. Charlson ME, Pompei $P$, Ales KL, MacKenzie CR. A new method of classifying prognostic comorbidity in longitudinal studies: development and validation. J Chronic Dis 1987; 40: 373-383

26. Quan H, Sundararajan V, Halfon P, Fong A, Burnand B, Luthi JC et al. Coding algorithms for defining comorbidities in ICD-9-CM and ICD-10 administrative data. Med Care 2005; 43: $1130-1139$.

27. Hankey GJ, Eikelboom JW, Ho WK, van Bockxmeer FM. Clinical usefulness of plasma homocysteine in vascular disease. Med J Aust 2004; 181: 314-318.

28. Holmes MV, Newcombe P, Hubacek JA, Sofat R, Ricketts SL, Cooper J et al. Effect modification by population dietary folate on the association between MTHFR genotype, homocysteine, and stroke risk: a meta-analysis of genetic studies and randomised trials. Lancet 2011; 378: 584-594.

29. Saposnik G, Ray JG, Sheridan P, McQueen M, Lonn E. Homocysteine-lowering therapy and stroke risk, severity, and disability: additional findings from the HOPE 2 trial. Stroke 2009; 40: 1365-1372.

30. Almeida OP, Alfonso H, Jamrozik K, Hankey GJ, Flicker L. Aspirin use, depression, and cognitive impairment in later life: the health in men study. J Am Geriatr Soc 2010; 58: 990-992.

31. Fiebich BL, Candelario-Jalil E, Mantovani M, Heinzmann M, Akundi RS, Hull M et al. Modulation of catecholamine release from rat striatal slices by the fixed combination of aspirin, paracetamol and caffeine. Pharmacol Res 2006; 53: 391-396.

32. Bottiglieri T, Laundy M, Crellin R, Toone BK, Carney MW, Homocysteine Reynolds EH. folate, methylation, and monoamine metabolism in depression. $J$ Neurol Neurosurg Psychiatry 2000; 69: 228-232.

33. Walker JG, Mackinnon AJ, Batterham P, Jorm AF, Hickie I, McCarthy A et al. Mental health literacy, folic acid and vitamin B12, and physical activity for the prevention of depression in older adults: randomised controlled trial. Br J Psychiatry 2010; 197: 45-54.

Translational Psychiatry is an open-access journal published by Nature Publishing Group. This work is licensed under the Creative Commons Attribution-NonCommercial-No Derivative Works 3.0 Unported License. To view a copy of this license, visit http://creativecommons.org/licenses/by-nc-nd/3.0/ 